\section{$\$$ Research Square}

\title{
Hyomental distance measured by ultrasound for size selection of laryngeal mask airway in female patients: a randomized controlled study
}

\section{xia wang}

Guangzhou University of Chinese Medicine

\section{Zhi-Hang Tang}

Guangzhou University of Traditional Chinese Medicine First Affiliated Hospital

wuhua ma ( $\sim$ tuesdaymorninggz@126.com )

The First Affiliated Hospital of Guangzhou University of Chinese Medicine, Guangzhou https://orcid.org/0000-0003-1277-1681

Research article

Keywords: laryngeal mask airway, Size, ultrasound

Posted Date: January 25th, 2021

DOI: https://doi.org/10.21203/rs.3.rs-152376/v1

License: (a) (1) This work is licensed under a Creative Commons Attribution 4.0 International License. Read Full License 


\section{Abstract}

\section{Background}

Optimal size selection of classic laryngeal mask airway (LMA) remains a major challenge for anaesthesiologists. Because the body weight, current method to select LMA size, might not inconsistent with dimension of hypopharynx where LMA is positioned. Hyomental distance by ultrasound as an common parameter in upper airway may be considered a predictor of LMA size choice, that we conducted a randomized, controlled study to compare the efficacy of size selection between commonly recommended weight-based method and hyomental distance-based method.

\section{Methods}

Seventy female patients undergoing breast cancer surgery were enrolled in our study and randomly assigned into either the hyomental distance group or weight group. The primary outcome was the correct rate of selection LMA without need for size adjustment or use of other devices. Secondary outcomes included injury rate, oropharyngeal leak pressure (OLP), and insertion attempts.

\section{Results}

Two participants were excluded from weight group due to tracheal intubation. The correct rate of LMA size-selected were significant difference of $77.14 \%$ and $51.51 \%$ in hyomental distance group and weight group, respectively $(\mathrm{P}=0.027)$. Five $(14.29 \%)$ and $7(21.21 \%)$ patients were injured in hyomental-distance group and weight group, respectively. Values of OLP in two groups were 22 (19-24) $\mathrm{cmH}_{2} \mathrm{O}$ and 22 (19.524) $\mathrm{cmH}_{2} \mathrm{O}$, respectively. The insertion attempts were the same in both groups and no statistical differences were observed regarding these three outcomes.

\section{Conclusion}

The hyomental distance-based method possessed a higher success rate for LMA-classic size selection than did the weight-based method, with the same excellent OLP value, low possibility of injury, and a low number of insertion attempts as weight-based method.

\section{Trial registration}

Our study was registered with the Chinese Clinical Trial Registry (ChiCTR1900021123), registered 29 January 2019.

\section{Background}

Laryngeal mask airway (LMA) as an alternative technique for airway management, due to its ease of application and its association with lower complication rates, is increasingly administered in elective surgery, resuscitation, and emergency scenarios involving airway complications [1-4]. Weight-based 
criteria for size selection has been routinely taught and recommended for clinical practice [5-6]. However, appropriate LMA size selection remains a major challenge for anaesthesiologists, as there is often a lack of correlation between an individual's upper airway anatomy and body weight[7].

A previous study demonstrated that one of upper airway anatomy parameters of hyomental distance, the distance between hyoid bone and tip of the chin, may considered a predictor for LMA size choice[8]. As such, we designed a prospective, randomised controlled study to compare the efficacy of weight-based method to hyomental distance-based method for individualized estimation of LMA size selection in breast cancer surgery patients.

\section{Methods}

\section{Ethics Statement}

Ethical approval was obtained from the First Affiliated Hospital of Guangzhou University of Chinese Medicine, Guangzhou, China (NO.ZYYECK[2018]041). Our study was registered with the Chinese Clinical Trial Registry (ChiCTR1900021123). All patients volunteered to take part in this study and provided written informed consent before being selected.

\section{Study Population}

We recruited patients between May 1, 2019 and August 31, 2019 who presented with American Society of Anesthesiologists (ASA) physical status I or II and were aged between 18-70 years. Inclusion and exclusion criteria are reported in Table 1.

Table 1. Trial inclusion and exclusion criteria

Inclusion Criteria Exclusion Criteria

Age: $18-70$ years

Patients weighing $<30 \mathrm{~kg}$ or $>75 \mathrm{~kg}$, pregnant women, and parturients

ASA grade I or II

Patients with a high risk of regurgitation or aspiration (i.e., large hiatal hernia, history of gastroesophageal reflux disease (GERD), or uncontrolled diabetes mellitus)

Patients undergoing elective breast cancer surgery under general anaesthesia

Patients with abnormal pharynx or anatomy, severe cardiovascular or cardiopulmonary diseases, hepatic or renal dysfunction, or coagulation disorders

Patients who agreed to the use

Patients who requested tracheal intubation during surgery of LMA-classic during surgery

Consent to participate

Refusal to participate 
Before study, we did a pilot experiment, measured the upper airway parameters of 10 subjects who using LMA during operation by ultrasound, we found that when the hyomental distance was greater than 4.41 $\mathrm{cm}, 80 \%$ of subjects were suit for class 4 LMA. So we hypothesized that if a patient's hyomental distance was at least $4.41 \mathrm{~cm}$ or their weight was greater than $50 \mathrm{~kg}$, the class 4 LMA would be selected for insertion, all other instances resulted in a class 3 selection $[9,10]$.

In this single-blind study, 70 patients were randomly assigned into two groups via selection of an envelope from a shuffled stack. Each patient selected an envelope immediately before surgery to assign them to one study group. Patients were not made aware of which study group they were assigned. The ultrasound parameters for hyomental distance were recorded for the hyomental distance group.

An anaesthesiologist proficient in ultrasound administration was responsible for measurement and data recording. Two other anaesthesiologists were responsible for LMA-classic selection and insertion.

Preoperative evaluation was performed the day before surgery. After routine fasting (no solids, $\geq 8 \mathrm{hr}$; no water, $\geq 4 \mathrm{hr}$ ), patients were sent to operating room (OR) and positioned supine on the operating table with their head in sniffing position [11,12]. Patients were subjected to pulse oximetry, noninvasive blood pressure monitoring (certain patients required invasive arterial monitoring), electrocardiography, and capnography. Before anaesthesia induction, the hyomental distance was measured by ultrasound (Navi series, Shenzhen Wisonic Medical Technologies,Shenzhen, China).

Patients were preoxygenated for 3 minutes through a facemask using $100 \%$ oxygen $(5-6 \mathrm{~L} / \mathrm{min})$. Then, moderate doses of remifentanil $(2 \mu \mathrm{g} / \mathrm{kg})$ and propofol $(2 \mathrm{mg} / \mathrm{kg})$ were given through intravenous infusion until patients lost consciousness, then, subsequently administered muscle relaxants of vecuronium bromide for each participants $(0.08-0.12 \mathrm{mg} / \mathrm{kg})$. The ventilation condition was determined by observing the end-tidal $\mathrm{CO}_{2}\left(\mathrm{EtCO}_{2}\right)$ curve. Once patient's condition was sufficient for LMA insertion (i.e., loss of eyelash reflex, jaw relaxation, or absence of movement), a selected lubricated LMA-classic (TUORen, HeNan, China) was inserted by an experienced doctor who had administered LMA-facilitated airway management in more than 1,000 prior cases. The LMA cuff was inflated with air until its pressure reached approximately $60 \mathrm{cmH}_{2} \mathrm{O}$ [13]. A senior anaesthesiologist then confirmed whether the selected LMA was properly sized for patients who should meeting all following criteria: (1) observation of slight outward movement of the LMA tube upon full cuff inflation, (2) smooth and regular visualization of exhaled $\mathrm{CO}_{2}$ on the capnograph, (3) obvious chest undulation without air leakage or stomach undulation, and (4) a fiberscope grading of more than 2 . Fiberscope grading was defined as: $4=$ only vocal cords visible, 3 = vocal cords and posterior epiglottis visible, 2 = vocal cords plus anterior epiglottis visible, and $1=$ vocal cords invisible $[9,14,15]$.

Values of oropharyngeal leak pressure (OLP) after airway management, duration of operation (the time elapsed from LMA insertion to removal), and postoperative oral injury (defined by blood adhering to the LMA surface) were detected and recorded for each patient. 
For patients' safety, if the chosen LMA which was confirmed by the senior anaesthesiologist was not suitable for the patient, we would replaced a more fitting LMA or performed tracheal intubation; such cases would be recorded.

\section{Ultrasonographic Measurement}

All ultrasound measurements were performed by a single researcher after positioning patients supine with head and neck in neutral position. A low frequency probe was placed on patient's neck perpendicular to skin to measure hyomental distance [8] (Figure 1). The researcher adjusted the ventilation according to the appropriate parameters for each patient. Patients were observed closely to prevent LMA displacement during the operation due to airway pressure. Anaesthesia was maintained with propofol and remifentanil.

Primary outcome was the correct rate of the predicted LMA size without needing for size adjustment or employing other devices. Secondary outcomes included injury rate (defined as visible blood stains on LMA upon removal), OLP value, and number of insertion attempts (defined as re-insertion after complete LMA removal from the oral cavity). The LMA adjustment process was not included as a measured secondary outcome.

\section{Statistical Analysis}

Based on results of previous studies [2ه13], the minimum sample size required to detect a significant difference is at least 32 in each group (64 in total) for the first attempt between two groups, considering type I error (alpha) of 0.05 , power (1-beta) of 0.8 , we decided to include 70 participants in order to meet experimental requirements. The Kolmogorov-Smirnov test was used to detect the normality of quantitative data. Based on test results, data were displayed as mean \pm standard deviation or median $\left(P_{25}-P_{75}\right)$-appropriately -while categorical data were presented as numbers. Pearson's chi-squared test was used to compare success rates of LMA size-selection between weight group and hyomental-distance group. Wilcoxon rank test of two independent samples was used to examine OLP values and other outcomes. IBM SPSS Version 25.0 software was used for statistical analysis. A P value less than 0.05 was used to determine significance.

\section{Results}

During observation period, 70 patients were consecutively enrolled. However, two participants from weight group were subsequently excluded due to a need for tracheal intubation. Overall, 35 patients in hyomental-distance group and 33 patients in weight group were involved in this study. The CONSORT flowchart of this trial is provided in figure 2 .

And the characteristics of all patients are displayed in Table 2.

Table 2. Comparison of patient characteristics between two groups 


\begin{tabular}{|c|c|c|c|}
\hline \multirow[t]{2}{*}{ Variable } & Hyomental-distance group & Weight group & $P$ \\
\hline & $\mathrm{N}=35$ & $\mathrm{~N}=33$ & Value \\
\hline $\operatorname{Age}^{\mathrm{a}}(\mathrm{yr})$ & $46.92 \pm 12.13$ & $52.06 \pm 8.011$ & 0.066 \\
\hline Weight $^{\mathrm{a}}(\mathrm{kg})$ & $58.5 \pm 9.531$ & $57.73 \pm 6.406$ & 0.854 \\
\hline Body mass index ${ }^{\mathrm{a}}\left(\mathrm{kg} / \mathrm{m}^{2}\right)$ & $22.71 \pm 3.419$ & $22.93 \pm 2.732$ & 0.731 \\
\hline Duration of LMA placement ${ }^{\mathrm{a}}(\mathrm{min})$ & $220.9 \pm 86.30$ & $198.5 \pm 42.65$ & 0.436 \\
\hline
\end{tabular}

Data are shown as mean \pm standard deviation, or numbers.

${ }^{a} A l l$ patient characteristics were compared using Mann-Whitney $U$ test for continuous variables.

\section{Primary Outcome Results}

Of all 68 participants, 27 participants from hyomental-distance group received accurately-selected LMAs, compared to 17 patients from weight group. The correct rates of the two groups were $77.14 \%$ and $51.51 \%$, respectively. A significant difference $(P=0.027)$ was observed between two methods (Figure $3 a)$.

\section{Secondary Outcome Results}

Five (14.29\%) hyomental-distance group patients and seven (21.21\%) weight group patients were injured(Figure 3b). OLP values for two groups were 22 (19-24) cmH2O and 22 (19.5-24) cmH2O, respectively (Figure 4a). Insertion attempts were the same for both groups (Figure 4b). No statistical differences were observed between two methods for each of three secondary outcomes (Table 3).

Table 3. Comparison of primary and secondary outcomes between groups

\begin{tabular}{|llll|}
\hline Variable & $\begin{array}{l}\text { Hyomental-distance group } \\
\mathrm{N}=35\end{array}$ & $\begin{array}{l}\text { Weight group } \\
\mathrm{N}=33\end{array}$ & $\begin{array}{l}P \\
\text { Value }\end{array}$ \\
\hline Successful rate (\%)† & 77.14 & 51.51 & $0.027^{*}$ \\
\hline Injury rate $(\%) \dagger$ & 14.29 & 21.21 & 0.454 \\
\hline OLP $\left(\mathrm{cmH}_{2} \mathrm{O}\right) \boldsymbol{\Lambda}$ & $22(19-24)$ & $22(19.5-24)$ & 0.082 \\
\hline Insertion attempts & $1(1-2)$ & $1(1-2)$ & 0.673 \\
\hline
\end{tabular}

${ }^{*} P<0.05$ compared with two groups

† Values compared using Pearson's chi-squared test 
\ Outcomes compared using Wilcoxon rank tests.

\section{Discussion}

In present study, we found hyomental distance might provide a higher correct rate of classic LMA size selection than did weight-based criteria for optimal size selection. Furthermore, when hyomental distance was more than $4.41 \mathrm{~cm}$, the prediction accuracy of LMA size selection was $77.14 \%$, the weight-based method only saw $51.51 \%$ accuracy $(P=0.027)$. This finding suggest that the hyomental distance-based method may provides for more optimal size selection of LMA-classic duringsurgery operations. Overall primary and secondary outcomes considered together, our study indicated that not only does hyomental distance-based method possess the higher size selection correct rate, but it also produces a similarly strong OLP value, low injury rate, and low number of insertion attempts when compared to the weightbased criterion.

As we know, anaesthesia is becoming more precise and visualized due to integration of various imaging techniques [16-18]. Point of care ultrasound (POCUS) -a portable, non-invasive, simple, and valuable tool for observing upper airway tissues-is increasingly used in airway management, even for anatomydistorted patients $[19,20]$. Several other selection methods have been described with varying results, including sex-based criteria [10] and cricoid-mental distance [13], these methods have not been shown to detect the internal airway anatomy for each patient, which is a crucial difference to reflect proper size selection of extraglottic airway devices [21]. However, sonographic measurement of hyomental distance offers a more precise and reliable method for classical LMA size selection by probing the upper respiratory tract.

In addition, LMA has been preferentially used in short and small surgery or first aid scenarios [22]. But, in recent years, because of easier operations with less postoperative complications, higher levels of comfort, and shorter recovery time than seen with endotracheal intubation [23], LMA is increasingly used in major operations, such as breast cancer surgery, limb fracture surgery, and even spinal surgery [24]. Breast surgery is one of the best indications for using LMA because such surgery often involves longer operation time, thus necessitating more significant requirement for accurate LMA size selection. Additionally, the procedure also did not involve use of laparoscopic pneumoperitoneum, which possibility of improper LMA positioning was relatively low. Therefore, it was the best choice for our study.

Moreover, Due to longer duration of operation in this study, appropriate amount of muscle relaxant used in our study differs from most other experiments in which muscle relaxants were not used to facilitate LMA insertion[3,13]. It's the same as studies by Guanghui An et al. and Kong $\mathrm{M}$ et al. showing that muscle relaxants may be beneficial for LMA placement and maintenance [22,25-26].

\section{Limitations}


As in previous studies involving sonographic measurements [27,28], our study was limited by its association with need for ultrasound measurement. The hyomental distance-based method may not be applicable in environments without an ultrasound system, such as outside of the operating room. However, with rapid development of ultrasonic instruments, an increasing amount of portable machines have been created to meet demand. This study was further limited by its investigation of LMA-classic insertion in female patients who weighing $30 \mathrm{~kg}$ to $75 \mathrm{~kg}$ alone, patients weighing more than $75 \mathrm{~kg}$ are not few, this restriction may weaken the generalization of the conclusion. More research taking males and children or obese subjects into account may be conducted in the future as the next step in our study. Finally, the results may differ for other types of LMA, such as LMA supreme or ProSeal LMA.

\section{Conclusion}

Our study demonstrated that the size selection of LMA-classic based on ultrasound measurement of hyomental distance provided a higher success rate of first insertion than that observed with the weightbased method. Both methods had the same airway seal and injury rate. The hyomental distance method provides a more precise visual method that may grow in popularity among anaesthesiologists.

\section{Abbreviations}

LMA: laryngeal mask airway; ASA: American Society of Anesthesiologists; OR: operating room; OLP: oropharyngeal leak pressure; POCUS: Point of care ultrasound.

\section{Declarations}

\section{Ethics approval and consent to participate}

This study was approved by the First Affiliated Hospital of Guangzhou University of Chinese Medicine, Guangzhou, China (NO.ZYYECK[2018]041) and registered at clinicaltrials.gov (identifier: ChiCTR1900021123.) Written informed consent was obtained from each patient.

\section{Consent for publication}

Not applicable.

\section{Availability of data and materials}

The datasets used during the current study are available from the corresponding author on reasonable request.

\section{Competing interests}

There are no conflicts of interest. 


\section{Funding}

This work was supported by NSFC General Projects(NO.81673922) granted from the National Natural Science Foundation of China, and the Science and Technology Program of Guangzhou (NO.201707010297) granted from Guangzhou Municipal Science and Technology Bureau. These funders provided the necessary financial aids during the whole study.

\section{Authors' contributions}

Study conception and design, acquisition of the data, analysis and interpretation of the data: XW, ZHT. Drafting of the manuscript: XW. Critical revision: WHM. All authors read and approved the final manuscript.

\section{Acknowledgements}

None.

\section{Author details}

1Guangzhou University of Chinese Medicine, Guangzhou, China

2Department of Anesthesia, The First Affiliated Hospital of Guangzhou University of Chinese Medicine, Guangzhou, China

\section{References}

1. Cook TM, Woodall N, Harper J, et al. Major complications of airway management in the UK: results of the Fourth National Audit Project of the Royal College of Anaesthetists and the Diffcult Airway Society. Part 2: intensive care and emergency departments. Br J Anaesth 2011; 106: 632-642.

2. Weng $M$, Ding $M, X u Y$, et al. An evaluation of thyromental distance-based method or weight-based method in determining the size of the laryngeal mask airway supreme: A Randomized Controlled Study. Medicine 2016; 95: 1-6.

3. Singh A, Bhalotr AR, Anand R. A comparative evaluation of Proseal laryngeal mask airway: A randomised trial. Indian J Anaesth 2018; 62: 858-864.

4. Rao M, Pillai A, Sharma J, et al. Comparison of lateral insertion technique of ClassicTM laryngeal mask airway along right versus left border of tongue in adults. Sri Lankan Journal of Anaesthesiology 2019; 27: 40-46.

5. Brimacombe J, Brain AlJ, Berry A. Non anesthetic uses. In: The laryngeal mask airway: a review and practice guide. Philadelphia, Pennsylvania: Saunders; 1997. pp. 216-277.

6. Zahoor A, Ahmd N, Sereche G, et al. A novel method to determine the size of the laryngeal mask airway in children. Eur J Anaesthesiol 2012; 29: 386-390.

7. The LMA supreme instruction manual. Mainenhead: Intravent Orthofix Ltd; 2007. 
8. Petri C, Dîrzu D, Trancă S, et al. Preoperative difficult airway prediction using suprahyoid and infrahyoid ultrasonography derived measurements in anesthesiology. Med Ultrason 2019; 21: 83-88.

9. Ozgul U, Erdil FA, Erdogan MA, et al. Comparison of videolaryngoscope-guided versus standard digital insertion techniques of the ProSeal ${ }^{T M}$ laryngeal mask airway: a prospective randomized study. BMC Anesthesiology 2019; 19: 244.

10. Kihara S, Brimacombe JR, Yaguchi Y. A Comparison of Sex- and Weight-based ProSeal ${ }^{\mathrm{TM}}$ Laryngeal Mask Size Selection Criteria. Anesthesiology 2004; 101: 340-343.

11. Mendonca C, Ungureanu N, Nowicka A, et al. A randomised clinical trial comparing the 'sniffing' and neutral position using channelled (KingVision $\AA$ ) and non-channelled (C-MAC $®$ ) videolaryngoscopes. Anaesthesia 2018; 73: 847-855.

12. Dong $F$, Wang $Y$, Wang $X$, et al. Changes in the upper airway following induction of anaesthesia: a prospective observational study protocol to determine the use of ultrasound in the assessment of a difficult airway in China [J]. BMJ Open 2019; 9: 1-7.

13. Zhu Y, Shen W, Yiquan Lin, et al. Cricoid-mental distance-based versus weight-based criteria for size selection of classic laryngeal mask airway in adults: a randomized controlled study. J Clin Monit Comput 2019; 33:759-765.

14. Brimacombe J, Berry A. A proposed fiber-optic scoring system to standardize the assessment of laryngeal mask airway position. Anesth Analg 1993; 76: 457.

15. Keller C, Brimacombe J, Puhringer F. A fibreoptic scoring system to assess the position of laryngeal mask airway devices. Interobserver variability and a comparison between the standard, flexible and intubating laryngeal mask airways. Anasthesiol Intensivmed Notfallmed Schmerzther 2000; 35: 692694.

16. Gupta K, Gupta PK. Assessment of difficult laryngoscopy by electronically measured maxillopharyngeal angle on lateral cervical radiograph: A prospective study [J]. Saudi J Anaesth 2010; 4: 158-162.

17. Nagamine $Y$, Kurahashi K. The use of three-dimensional computed tomography images for anticipated difficult intubation airway evaluation of a patient with Treacher Collins syndrome [J]. Anesth Analg 2007; 105: 626-628》

18. Kundra P, Mishra SK, Ramesh A. Ultrasound of the airway [J]. Indian J Anaest 2011; 55: 456-462.

19. Osman A, Sum KM. Role of upper airway ultrasound in airway management. J Intensive Care 2016; 4: 52.

20. Kim J, Kim JY, Kim WO. An Ultrasound Evaluation of Laryngeal Mask Airway Position in Pediatric Patients: An Observational Study. Anesth Analg 2015; 120: 427-432.

21. Cattano D, Zundert TV, Wojtzack J, et al. A new method to test concordance between extraglottic airway device dimensions and patient anatomy. Anesthesiology 2014; A3148.

22. An G, Fang B, Wang Z. Comparing the insertion and ventilation of laryngeal mask airway according to the patient's head position and muscle relaxation use. Saudi Med J 2019; 40: 687-693. 
23. Du X, Mao S, Cui J, et al. Use of laryngeal mask airway for non-endotracheal intubated anesthesia for patients with pectus excavatum undergoing thoracoscopic Nuss procedure. J Thorac Dis 2016; 8: 2061-2067.

24. Kang F, Li J, Chai XQ, et al. Comparison of the I-gel Laryngeal Mask Airway With the LMA-Supreme for Airway Management in Patients Undergoing Elective Lumbar Vertebral Surgery. J Neurosurg Anesthesiol 2015; 27: 37-41.

25. Fujiwara A, Komasawa N, Nishihara I, et al. Muscle relaxant effects on placement efficacy of the laryngeal mask ProSeal ${ }^{\circledR}$ in anesthetized patients: a prospective randomized controlled trial. $J$ Anesth 2015; 29: 580-584.

26. Kong M, Li B, Tian Y. Laryngeal mask airway without muscle relaxant in femoral head replacement in elderly patients. Exp Ther Med 2016; 11: 65-68.

27. NK Yadav, P Rudingwa, Mishra SK, et al. Ultrasound measurement of anterior neck soft tissue and tongue thickness to predict difficult laryngoscopy. An observational analytical study [J]. Indian J Anaesth 2019; 63: 629-638.

28. Pinto J, Cordeiro L, Pereira C, et al. Predicting difficult laryngoscopy using ultrasound measurement of distance from skin to epiglottis [J]. J Crit Care 2016; 33: 26-31.

\section{Figures}
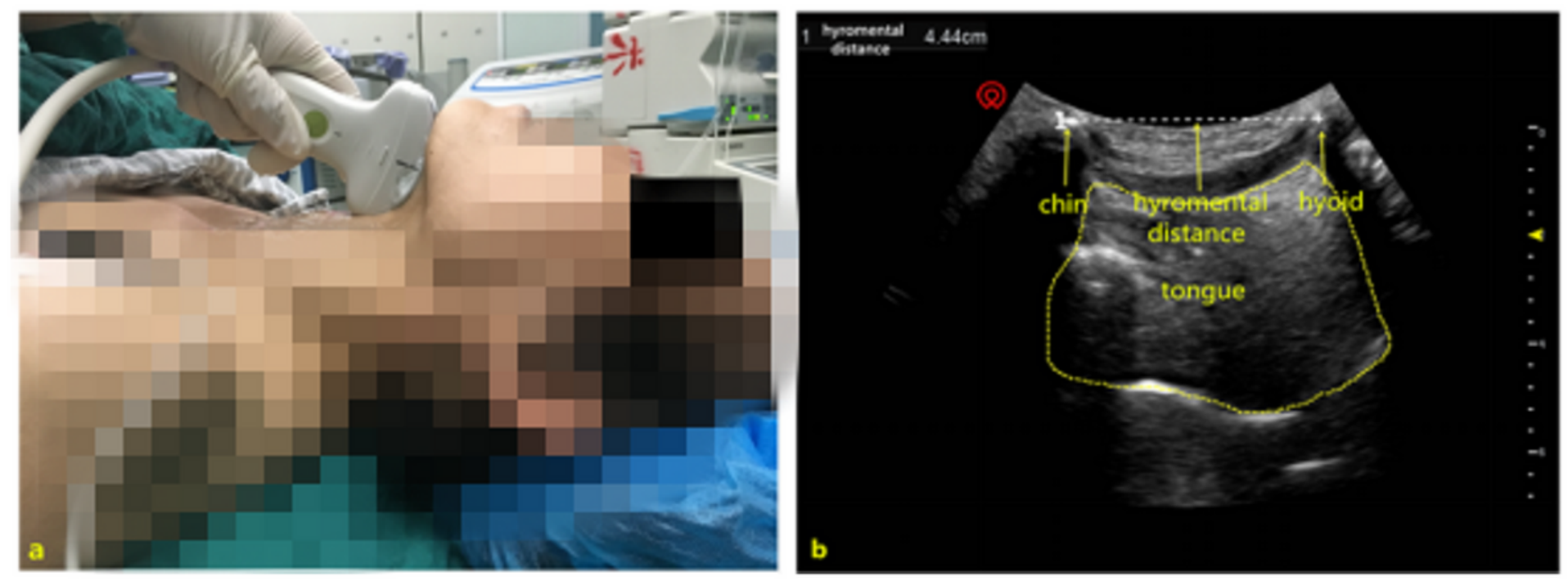

\section{Figure 1}

Hyomental distance measured by ultrasound. Ultrasonography axial views at the level of hyomental distance. A curved probe was placed parallel to the long axis of the body vertically in the median submental area (a). The Mark point was aligned to the mandible chin, and the other end of the probe was closely to the mandible skin. Then the chin, hyoid and tongue will appear on the ultrasound screen (b). The hyomental distance is defined as the straight-line between the chin and hyoid. 


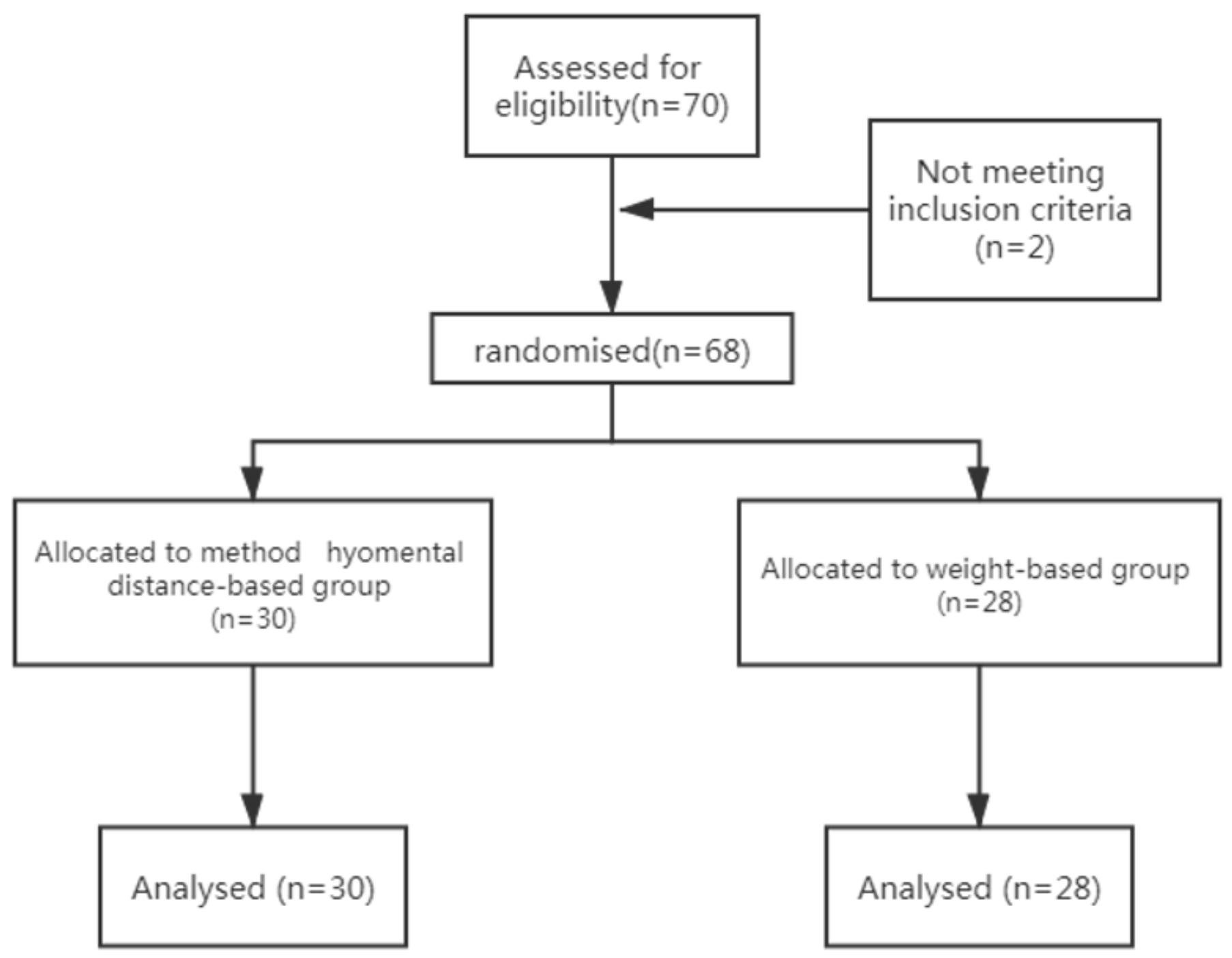

Figure 2

The CONSORT flowchart of study.

correct rate of two methods

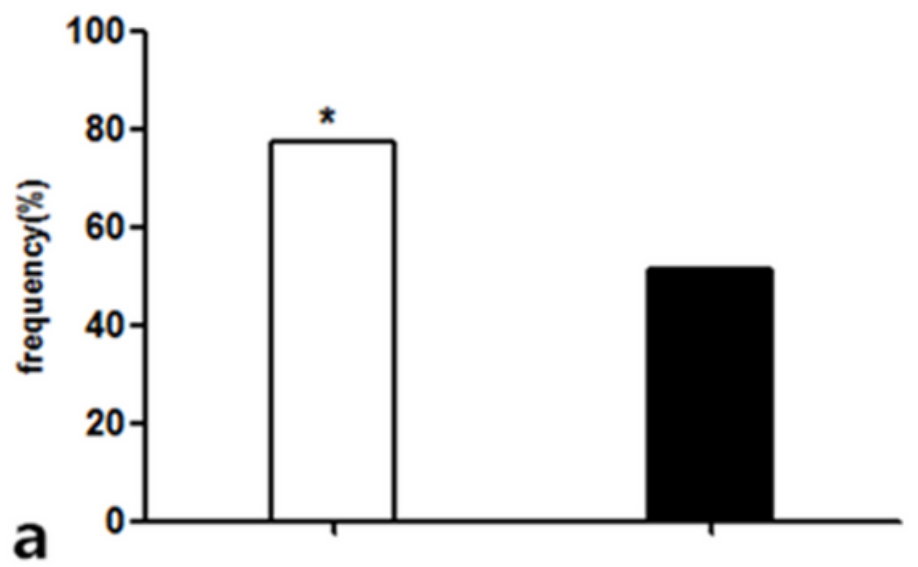

rate of injury with two methods

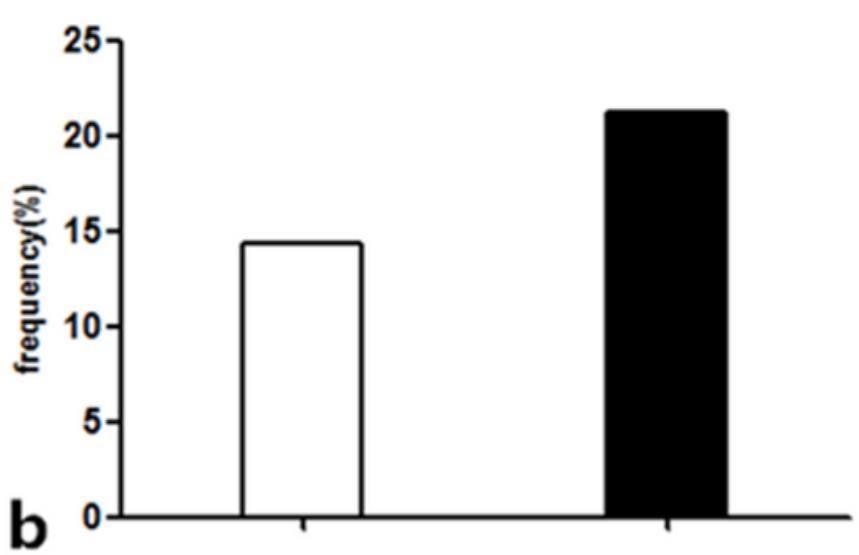

hyromental-distance group weight group 
Figure 3

The results of correct rate and injury between two method. The rate of correct and injury with two method were showed on picture $a$ and $b .{ }^{*} \mathrm{P}<0.05$ compared with two groups.
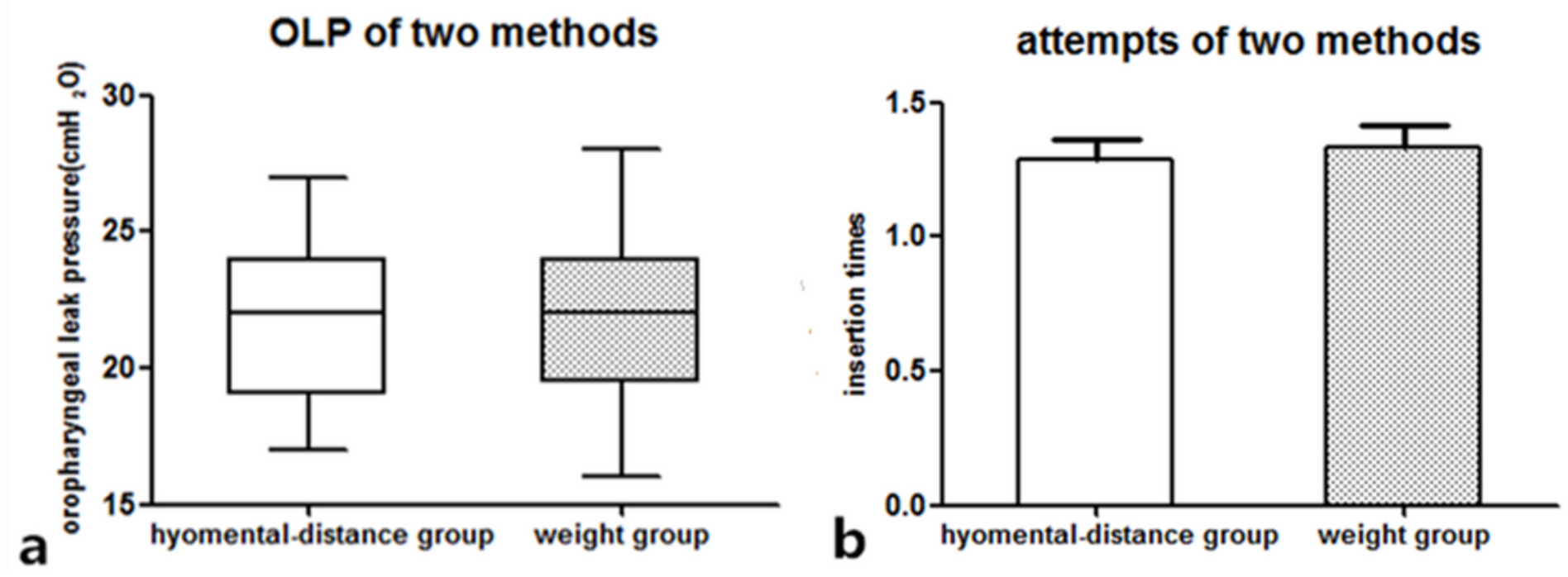

Figure 4

The box-plots images of OLP and attempts between two methods.

\section{Supplementary Files}

This is a list of supplementary files associated with this preprint. Click to download.

- CONSORTchecklist.docx 